\title{
Contributions of Pathway and Neuron to Preferential Motor Reinnervation
}

\author{
Thomas M. Brushart,, ${ }^{1,2}$ Jonathan Gerber, ${ }^{2}$ Philip Kessens, ${ }^{2}$ You-Gang Chen, ${ }^{1}$ and Richard M. Royall ${ }^{3}$ \\ ${ }^{1}$ The Raymond M. Curtis Hand Center, Union Memorial Hospital, Baltimore, Maryland 21218, ${ }^{2}$ Departments of \\ Orthopaedic Surgery and Neurology, Johns Hopkins School of Medicine, Baltimore, Maryland 21287, and ${ }^{3}$ Department \\ of Biostatistics and Statistics, School of Hygiene and Public Health, Johns Hopkins University, Baltimore, Maryland 21287
}

\begin{abstract}
Motor axons regenerating after transection of mixed nerve preferentially reinnervate distal muscle branches, a process termed preferential motor reinnervation (PMR). Motor axon collaterals appear to enter both cutaneous and muscle Schwann cell tubes on a random basis. Double-labeling studies suggest that PMR is generated by pruning collaterals from cutaneous pathways while maintaining those in motor pathways (the "pruning hypothesis"). If all collaterals projecting to muscle are saved, then stimulation of regenerative sprouting should increase specificity by increasing the number of motoneurons with at least one collateral in a muscle pathway. In the current experiments, collateral sprouting is stimulated by crushing the nerve proximal to the repair site before suture, a maneuver that also conditions the neuron and predegenerates the distal pathway. Control experiments are performed to separate these effects from those of collateral generation.

Experiments were performed on the rat femoral nerve and evaluated by exposing its terminal cutaneous and muscle
\end{abstract}

branches to HRP or Fluoro-Gold. Crush proximal to the repair site increased motor axon collaterals at least fivefold and significantly increased the percentage of correctly projecting motoneurons, consistent with the pruning hypothesis. Conditioning the nerve with distal crushes before repair had no effect on specificity. A graft model was used to separate the effects of collateral generation and distal stump predegeneration. Previous crush of the proximal femoral nerve significantly increased the specificity of fresh graft reinnervation. Stimulation of regenerative collateral sprouting thus increased PMR, confirming the pruning hypothesis. However, this effect was overshadowed by the dramatic specificity with which predegenerated grafts were reinnervated by fresh uncrushed proximal axons. These unexpected effects of predegeneration on specificity could involve a variety of possible mechanisms and warrant further study because of their mechanistic and clinical implications.

Key words: predegeneration; specificity; regeneration; nerve graft; motoneuron; conditioning
Preferential motor reinnervation (PMR) is the tendency for motor axons regenerating in mixed nerve to selectively reinnervate muscle (Brushart, 1988) or muscle nerve (Brushart, 1993). PMR has been observed by others (Madison et al., 1996; Neumann et al., 1996) and appears to be a constant feature of motor axon regeneration. The mechanism of PMR has been investigated in the rat femoral nerve (Brushart, 1990, 1993). Proximally, at the site of repair, cutaneous and muscle axons intermingle so that regenerating motor axons will have access to Schwann cell tubes leading to both muscle and skin. The accuracy of regeneration is assessed distally, where the nerve bifurcates into the branch to the quadriceps muscle and the saphenous nerve.

During early regeneration of the transected rat femoral nerve, equal numbers of motoneurons project exclusively to cutaneous or muscle nerves, while a third group projects collateral sprouts to both pathways simultaneously (Brushart, 1990). The number of motoneurons projecting correctly to muscle then increases dramatically, whereas the number projecting collaterals to both cutaneous and muscle pathways decreases. There is little change in the number of motoneurons projecting solely to skin. These

Received June 2, 1998; revised July 27, 1998; accepted Aug. 17, 1998

This work was supported by National Institutes of Health Grant R01 NS3448401A2 and the Raymond M. Curtis Research Fund. We thank Dr. J. Griffin for comments on this manuscript, K. Weaver for preparation of artwork, and Dr. P. Talalay for editorial assistance.

Correspondence should be addressed to Thomas M. Brushart, Johns Hopkins Orthopaedics, 601 North Caroline Street, Baltimore, MD 21287-0882.

Copyright (C) 1998 Society for Neuroscience $0270-6474 / 98 / 188674-08 \$ 05.00 / 0$ observations suggested the "pruning hypothesis" (Brushart, 1993): regenerating motor axons generate multiple collateral sprouts, which reinnervate previously sensory or motor Schwann cell tubes on a random basis. Over time, specific projections are generated by pruning collaterals from cutaneous pathways while maintaining those in muscle pathways. A motoneuron that initially samples both pathways is thus converted to one projecting correctly and only to muscle. Motoneurons limited to cutaneous pathways have no means of correcting their error, and their number remains relatively constant. In the absence of selective pruning, collateral projections to muscle and cutaneous nerve would be eliminated randomly, resulting in the loss of many correct muscle projections and ending with equal numbers of motoneurons projecting to skin and muscle. This outcome has not been observed in the course of multiple experiments.

The present experiments test the pruning hypothesis by examining a direct corollary. Increasing the number of collaterals generated by each motoneuron should improve the odds that at least one collateral will enter a muscle pathway. If every motoneuron with at least one collateral projection within muscle nerve is converted to a neuron projecting only to muscle, then increasing regenerative collateral sprouting should increase specificity. In these experiments, regenerative collateral sprouting was stimulated by nerve crush proximal to the repair site (see Fig. 1). When compared with routine nerve repair, this maneuver increased the percentage of motoneurons projecting to the quadriceps muscle by decreasing the number projecting to skin. Identical prepara- 


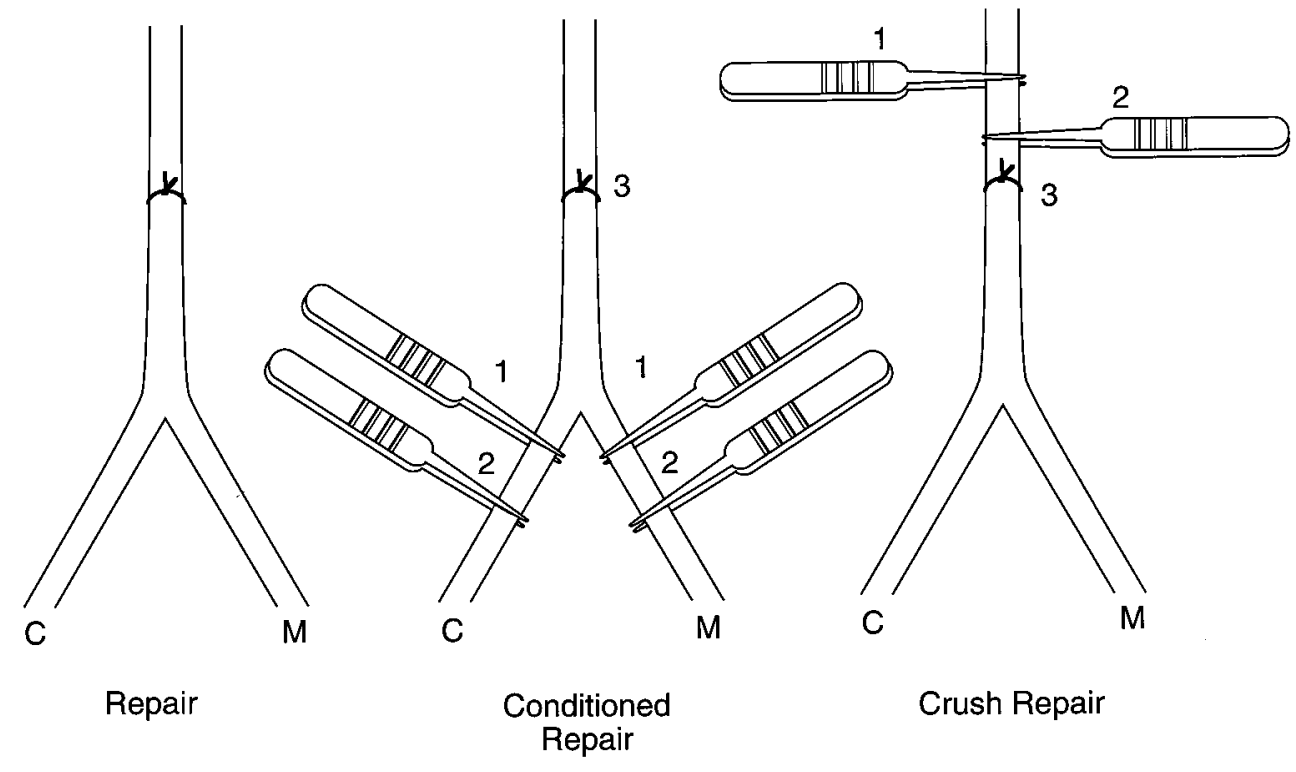

Figure 1. Preparation of nerve repairs. Surgery was performed on the proximal femoral nerves of $250 \mathrm{gm}$ female Sprague Dawley rats. In the repair group, the nerve was sharply transected and sutured under $20-40 \times$ with two $11-0$ sutures. In the conditioned repair group, the distal motor and sensory branches were each crushed for $5 \mathrm{sec}$ with \#5 jeweler's forceps. The crushes were administered 4 and 2 weeks before transection and suture of the proximal nerve. This preparation conditioned the neuron without stimulating collateral formation at the repair site. In the crush repair group, the proximal nerve was crushed, and after 2 weeks a second crush was delivered $2 \mathrm{~mm}$ distal to the first. After an additional 2 weeks, the nerve was repaired $2 \mathrm{~mm}$ distal to the second crush. This preparation maximized the number of axon collaterals at the suture site. $M$, Muscle projections; $C$, cutaneous projections. tions were thus investigated with a different labeling strategy (sequential double labeling) to determine the fate of motoneurons no longer projecting to skin after proximal crush. Nerve crush also conditions the neuron and predegenerates the distal pathway, so further experiments were performed to separate these effects from those of collateral generation. The effects of conditioning were isolated by performing sequential nerve crushes distal rather than proximal to the repair site (see Fig. 1). We designed a graft model to separate the effects of collateral generation and pathway predegeneration. Previously undamaged axons were directed to predegenerated grafts, and previously crushed axons were directed to fresh grafts.

\section{MATERIALS AND METHODS}

Nerve repair. Experiments were performed on both femoral nerves of young adult $(250 \mathrm{gm})$ female Sprague Dawley rats under Chloropent anesthesia ( $3 \mathrm{ml} / \mathrm{kg}$; Dodge Laboratories). Three types of repair were performed (Fig. 1). The "repair" group ( $n=60$ nerves) established a norm for evaluation of experimental manipulations. The proximal femoral nerve was sharply transected, carefully aligned, and sutured with 11-0 nylon under 20-40× magnification. The "crush repair" group $(n=$ 60 nerves) was designed to maximize the number of motor axon collaterals innervating the distal stump. The nerve was vigorously crushed with \#5 jeweler's forceps for $5 \mathrm{sec}$ so that the crushed segment became transparent. The first crush was delivered $4 \mathrm{~mm}$ proximal to the eventual repair site, and the second, 2 weeks later, was delivered $2 \mathrm{~mm}$ proximal to the repair site to reinjure the sprouts originating from the first crush. After an additional 2 weeks, the nerve was transected and sutured as in the repair group. In the "conditioning repair" group ( $n=40$ nerves), the distal femoral cutaneous and muscle branches were crushed 4 and 2 weeks before proximal suture to provide conditioning nerve injuries in the same time sequence but without stimulating collateral sprouting proximal to the repair site. Twenty nerves in each group were evaluated 3 weeks after suture, the index procedure, and an additional 20 were evaluated after 3 months, using a technique of simultaneous double labeling of projections to cutaneous and muscle nerve. In addition, 20 nerves from both repair and crush repair groups were evaluated after 3 months with sequential double labeling to determine the fate of motoneurons missing from the cutaneous nerve after crush repair.

Nerve grafting. Nerve grafting was performed to isolate the effects of collateral sprout generation and pathway predegeneration. The "graft" group ( $n=20$ nerves) established a baseline for the other grafting experiments by reinnervating a fresh graft with previously uninjured axons. The femoral nerve trunk and branches were excised and sewn into the bed of the opposite femoral nerve, correctly aligning muscle and cutaneous branches (see Fig. 5). In the "predegenerated graft" group
( $n=20$ nerves), the graft was predegenerated by crush 4 and 2 weeks before transfer and then transposed to the unoperated limb to be reinnervated by fresh, previously uninjured axons. This sequence was reversed in the "crush graft" group $(n=20$ nerves); the crushes were delivered to the recipient nerve to stimulate collateral sprouting, after which a fresh graft was transferred to receive these sprouts. All graft experiments were evaluated by simultaneous double labeling of cutaneous and muscle branches after 3 months of regeneration.

Control surgeries. Control experiments were performed to see if regenerated motor axons could transport HRP equally well from cutaneous and muscle pathways. The quadriceps and cutaneous femoral branches were transected at their origin from the femoral nerve. The quadriceps branch was repaired directly $(n=8)$ or transposed to reinnervate the cutaneous branch $(n=7)$. After 8 weeks of regeneration, HRP was applied to the distal end of the reinnervated branch, and spinal cord tissue was processed and evaluated to determine the number of labeled motoneurons. Additional control experiments were designed to assess the effects of proximal (crush repair, crush graft) and distal (conditioning repair) crushes on the number of axons at the repair site. The number of motor axon collaterals formed by sequential proximal crush was determined in the "pure motor model". The dorsal root ganglia serving the femoral nerve were excised unilaterally in 20 rats. Ten femoral nerves were harvested after 1 week to determine the number of motor axons at the normal repair site; the other 10 were subjected to proximal crush 4 and 2 weeks before harvest. To assess the effects of distal crush, four proximal femoral nerves were harvested after distal crush in the sequence and location used in conditioning repairs and compared with six previously unoperated femoral nerve specimens.

Simultaneous and sequential double labeling of motoneurons. One hundred eighty nerves were evaluated by simultaneous double labeling. This technique separately identifies motoneurons projecting correctly to the quadriceps muscle, incorrectly to the saphenous nerve, or simultaneously to both (double-labeled). The femoral cutaneous and muscle branches were exposed bilaterally. The muscle branch was severed as it entered the quadriceps muscle; the cutaneous branch was cut an equivalent distance from the femoral bifurcation to produce proximal cutaneous and muscle stumps of equal length. One stump (randomly chosen) was exposed to $10 \%$ HRP (Sigma VI; Sigma, St. Louis, MO) for $1 \mathrm{hr}$ in a Vaseline well, after which it was copiously irrigated and loosely sutured to a distant portion of the wound. The other stump was then exposed to 3\% FluoroGold (FG) (Schmued and Fallon, 1986; Brushart, 1990) for 2 hr, similarly irrigated, and sewn to the opposite corner of the wound to prevent cross-contamination by diffusion of tracers. Forty-eight hours were allowed for proximal transport of the tracers. Sequential double labeling was applied to 20 repair and 20 crush repair animals to determine the fate of motoneurons that projected to cutaneous nerve after repair but not after crush repair. The protocol for sequential double labeling differed only in the timing and location of exposure to tracers (see Fig. 3). 
Figure 2. Results of nerve repair. Each triad of vertical bars represents the mean motoneuron count obtained in 20 nerve preparations. The white bars represent the mean number of motoneurons projecting correctly to the muscle branch, the black bars represent the mean number projecting incorrectly to the cutaneous branch, and the stippled bars represent the mean number of double-labeled neurons, which project collaterals simultaneously to both cutaneous and muscle branches. In repair and conditioned repair groups, motoneuron projections were random at 3 weeks; PMR was clearly evident by 3 months. After crush repair, however, specificity was already apparent at 3 weeks and was dramatic by 3 months. This specificity was achieved by lowering the number of incorrect projections rather than by increasing the number of motoneurons projecting correctly to quadriceps muscle.

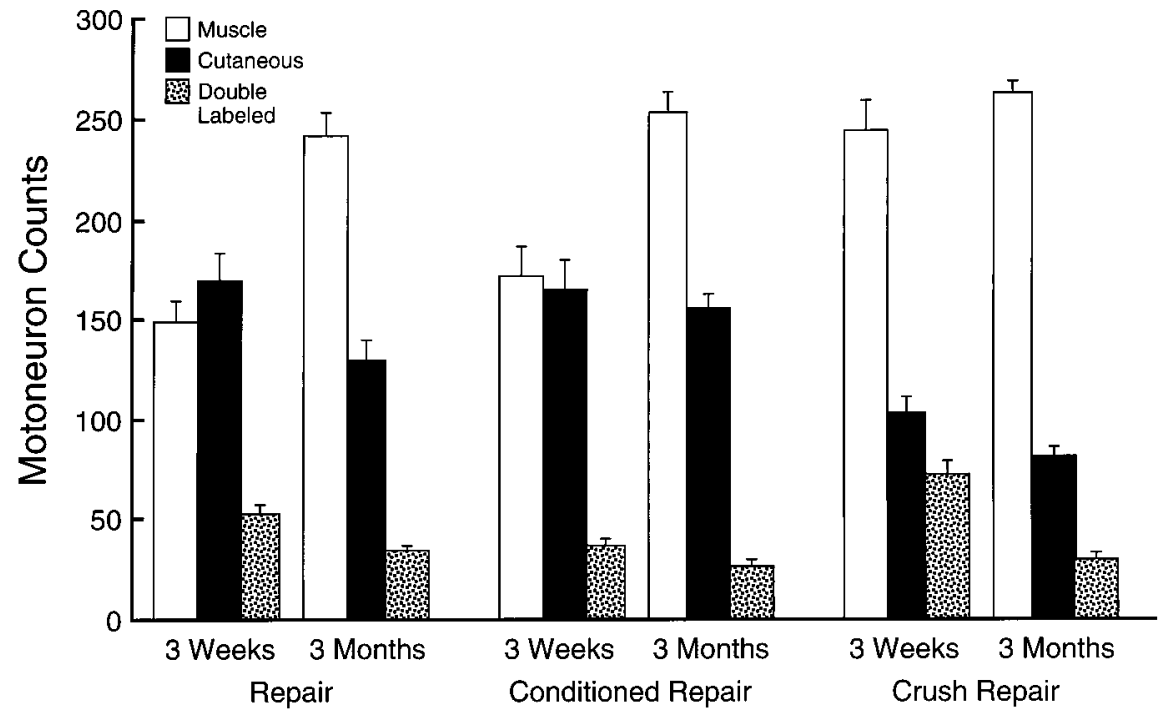

Initially, the cutaneous nerve was transected distally and exposed to either HRP or FG, randomly determined. Forty-eight hours were allowed for this tracer to label motoneurons projecting incorrectly to skin. The femoral trunk was then transected at the level of the iliacus muscle branch to label all motoneurons regenerating to the level of the iliacus muscle, and an additional $48 \mathrm{hr}$ were allowed for transport of the second tracer.

The animals were deeply anesthetized before perfusion through the left ventricle. A warm saline flush $(150 \mathrm{ml})$ was followed by the following: $500 \mathrm{ml}$ of $4 \%$ paraformaldehyde in $0.1 \mathrm{~m}$ sodium acetate buffer, $\mathrm{pH} 6.5$, over a half-hour; $500 \mathrm{ml}$ of $4 \%$ paraformaldehyde in $0.1 \mathrm{M}$ sodium borate buffer, $\mathrm{pH} 9.5$, over a half-hour; and $500 \mathrm{ml}$ of $10 \%$ sucrose in $0.1 \mathrm{M}$ Sorensen's phosphate buffer, $\mathrm{pH}$ 7.4, over a half-hour (Berod et al., 1981; Mesulam, 1982). The lumbar spinal cords were removed, and serial cross-sections were cut at $80 \mu \mathrm{m}$ on a freezing microtome. These sections were reacted with $\mathrm{H}_{2} \mathrm{O}_{2}$ and tetramethyl benzidine to demonstrate HRP within motoneurons (Mesulam, 1982). Sections were serially mounted on glass slides, dried, and coverslipped without counterstain to minimize background fluorescence.

Motoneuron counting and data analysis. Each spinal cord section was viewed with fluorescent ( $405 \mathrm{nM}$ ) and transmitted light at $20-40 \times$ by an observer unaware of which tracer had been used on which femoral branch. In evaluating the results of simultaneous double labeling, each femoral motoneuron pool was counted for the following: (1) HRPlabeled motoneurons; (2) FG-labeled motoneurons; and (3) doublelabeled motoneurons. The presence of split cells in adjacent sections was corrected for by the method of Abercrombie (1946). Each group was then characterized by three means: the mean number of motoneurons projecting correctly to the muscle branch; the mean number projecting incorrectly to the cutaneous branch; and the mean number of doublelabeled neurons, which project axon collaterals to both branches. A standard two-sample $t$ test was used to compare counts of motoneurons projecting to cutaneous and muscle branches within each group; a Welch modified two-sample $t$ test compared both the percentage of regenerating motoneurons and the absolute number of motoneurons labeled from the muscle branch across all six groups at 3 months. Both the percentage of double-labeled motoneurons and the percentage reinnervating the muscle branch were compared between 3 week and 3 month time intervals in repair, crush repair, and conditioned repair groups.

The results of sequential double labeling of repair and crush repair animals were evaluated by compiling means of the following: (1) the number of double-labeled motoneurons, which project to the cutaneous branch; and (2) the number of single-labeled motoneurons, which project correctly to muscle. The sum of these counts represented the total number of regenerating motoneurons. Similar $t$ test analyses were used to compare counts within and among groups.

Axon counting. Specimens taken from pure motor preparations to evaluate the effects of proximal crush were embedded in Epon-Araldite, thin sectioned, and viewed at $3000 \times$ through the electron microscope. Grids from the center and the four quadrants of the nerve were systematically identified and photographed. Counts of both myelinated and unmyelinated axons were then corrected for the total area of the nerve. Specimens of femoral nerve taken from the repair site to evaluate the effects of distal crush were embedded in Epon-Araldite, sectioned at $1 \mu \mathrm{m}$, counterstained with toluidine blue, and mounted on glass slides. All myelinated axons were counted and digitized with a Bioquant OS-2 system interfaced with a Nikon Optiphot microscope.

\section{RESULTS}

\section{Nerve repair}

The results of nerve suture are summarized in Figures 2 and 4. In the repair group, end-to-end suture in young adult rats produced results similar to those obtained previously in juveniles (Fig. 2) (Brushart, 1990, 1993). Three weeks after surgery, motor axon regeneration was random [quadriceps muscle projections (M), 149; cutaneous projections (C), 170; $p=0.24$ ]. After 3 months, however, significantly more motoneurons projected to muscle than to skin (M, 242; C, 130; $p=0.0001)$. The mean number of double-labeled motoneurons, which simultaneously project collaterals to both cutaneous and muscle branches, diminished from 53 at 3 weeks to 34 at 3 months $(p=0.0001)$. Conditioned repairs were similar to the repair animals, with no significant differences time period. In sharp contrast, the results of crush repair differed strikingly from those of the other groups. Three weeks after suture, dramatic specificity was already apparent (M, 244; C, 103; $p=0.0001)$. This difference was even greater at 3 months $(\mathrm{M}$, 263; C, $81 ; p=0.0001)$.

The percentage of motoneurons projecting exclusively to muscle $(\% \mathrm{M})$ is a conservative expression of motor-sensory specificity, because it takes into account double-labeled motoneurons in addition to those projecting exclusively to muscle or skin. In the repair group, specificity increased from $40 \% \mathrm{M}$ at 3 weeks to $60 \% \mathrm{M}$ at 3 months $(p=0.0001)$. In contrast, the crush repairs had already reached $58 \% \mathrm{M}$ at 3 weeks and increased to $70 \% \mathrm{M}$ at 3 months ( $p=0.0001$ between repair and crush repair groups at both 3 weeks and 3 months). Crush repair also increased the mean number of double-labeled motoneurons at 3 weeks from 53 in the repair group to 72 after crush repair $(p=0.02)$. Crush repair thus increased collateral sprouting, the substrate of pruning, and resulted in both earlier and more dramatic motorsensory specificity than was seen in either repair or conditioned repair groups. Nevertheless, the absolute number of motoneurons in the number of labeled motoneurons in either pathway at either 


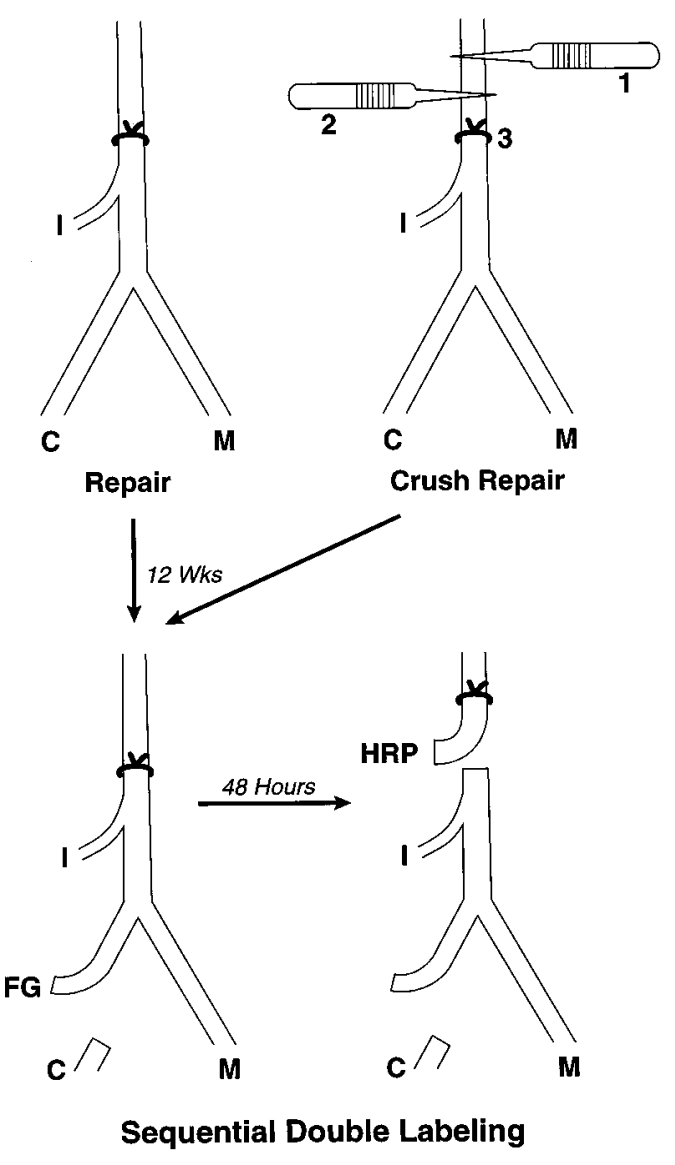

Figure 3. Sequential double labeling. Repair and crush repair animals were prepared as in Figure 2. After 3 months of regeneration, the cutaneous nerve was exposed to tracer, in this case FG, to label all motoneurons misdirected to skin. Forty-eight hours later, the proximal nerve was labeled with a different tracer, HRP, to label all motoneurons regenerating past the repair to the level of the iliacus nerve. Motoneurons projecting to skin will be double-labeled, and those projecting to muscle will be labeled with only the second tracer.

projecting correctly to the quadriceps muscle, the ultimate substrate of muscle function, did not differ significantly between crush repair and repair $(p=0.109)$ or conditioned repair ( $p=$ 0.48 ) groups. Specificity was thus increased by decreasing the number of motoneurons projecting incorrectly to skin, either by rerouting them to an unlabeled destination or by preventing their regeneration altogether.

Additional repair and crush repair animals were evaluated with sequential double labeling at 3 months to determine the fate of motoneurons projecting to skin after repair but not after crush repair. The simultaneous double-labeling technique treats cutaneous and quadriceps nerves as a well matched pair of terminal branches, neglecting the more proximal iliacus muscle branch and the minute branch to the pectineus muscle (Brushart, 1988, 1990). In contrast, sequential double labeling (Fig. 3) identifies motoneurons projecting to the cutaneous nerve in the context of all motoneurons regenerating past the repair site to the level of the iliacus muscle. The mean number of motoneurons projecting to skin was reduced from 143 after repair to 65 after crush repair ( $p=0.0001)$, whereas the mean total number of motoneurons regenerating was 651 in both groups (Fig. 4). Crush repair thus improved total muscle reinnervation by rerouting incorrect cuta-

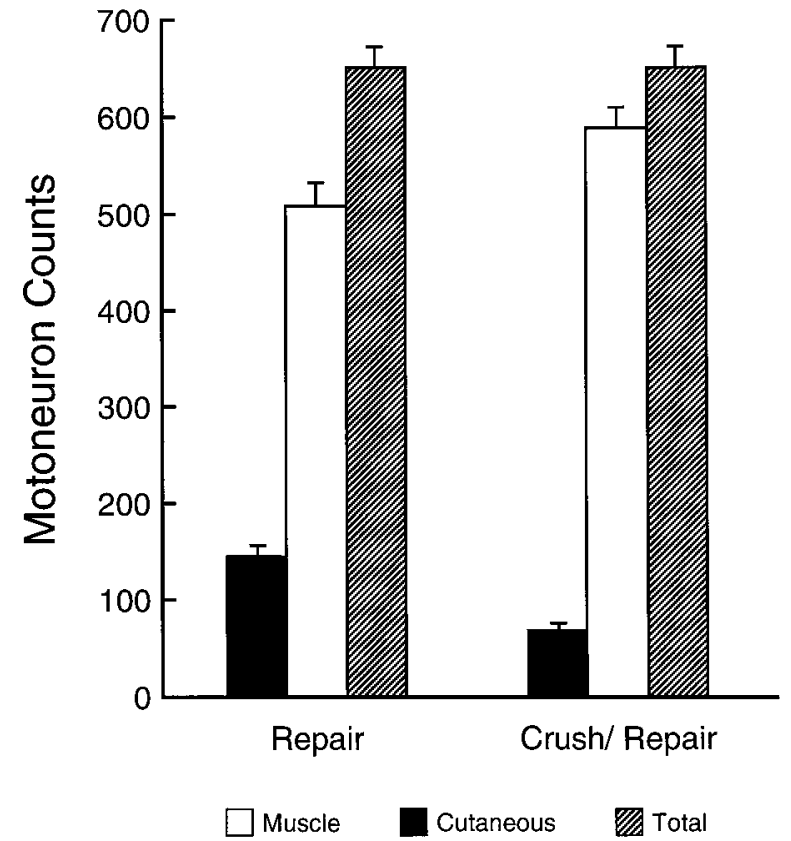

Figure 4. Results of sequential double labeling. Each triad of vertical bars represents the mean counts from 20 nerve preparations. The black bars represent the mean number of double-labeled motoneurons, which project incorrectly to skin, the white bars represent motoneurons projecting correctly to muscle, and the hatched bars represent the total number of motoneurons regenerating. Crush repair shunted motor axons from cutaneous to muscle nerve without decreasing the total volume of regeneration.

neous projections rather than by preventing motoneuron regeneration.

\section{Nerve grafting}

The predegenerated graft group (Fig. 5) was prepared to isolate the effects of distal pathway predegeneration inherent in the crush repair process, whereas the crush graft group isolated the effects of augmented collateral sprouting. Graft animals were prepared to control for the effects of graft transposition, devascularization, and the necessity for regenerating axons to cross two suture lines. As expected, fewer motoneurons reached the periphery after graft (mean, 320) than after repair (mean, 406; $p=0.0067$ ) (Fig. 6). However, motor-sensory specificity as expressed in the $\% \mathrm{M}$ remained constant (repair, $60 \% \mathrm{M}$; graft, $58 \% \mathrm{M} ; p=0.64$ ). Crush graft increased specificity with a \% $\mathrm{M}$ of $66(p=0.0097)$ yet did not increase the total number of motoneurons projecting to muscle (graft, 186; crush graft, 188; $p=0.9$ ). This increase in specificity by reducing cutaneous projections is analogous to the relationship between repair and crush repair discussed above. Results in the predegenerated graft group, however, were strikingly superior to those of routine graft. Specificity increased from $58 \% \mathrm{M}$ to $69 \% \mathrm{M}(p=0.001)$, and the total number of motoneurons reinnervating quadriceps muscle increased from 186 to 245 $(p=0.0018)$. These outstanding results were comparable to those of crush repair, both in terms of specificity (crush repair, $70 \% \mathrm{M}$; predegenerated graft, $69 \% \mathrm{M} ; p=0.48$ ) and mean number of motoneurons reinnervating the quadriceps (crush repair, 263; predegenerated graft, 245; $p=0.218$ ).

\section{Control experiments}

A mean of 380 motoneurons was labeled after the quadriceps muscle branch was self-reinnervated $(n=8)$ compared with a 

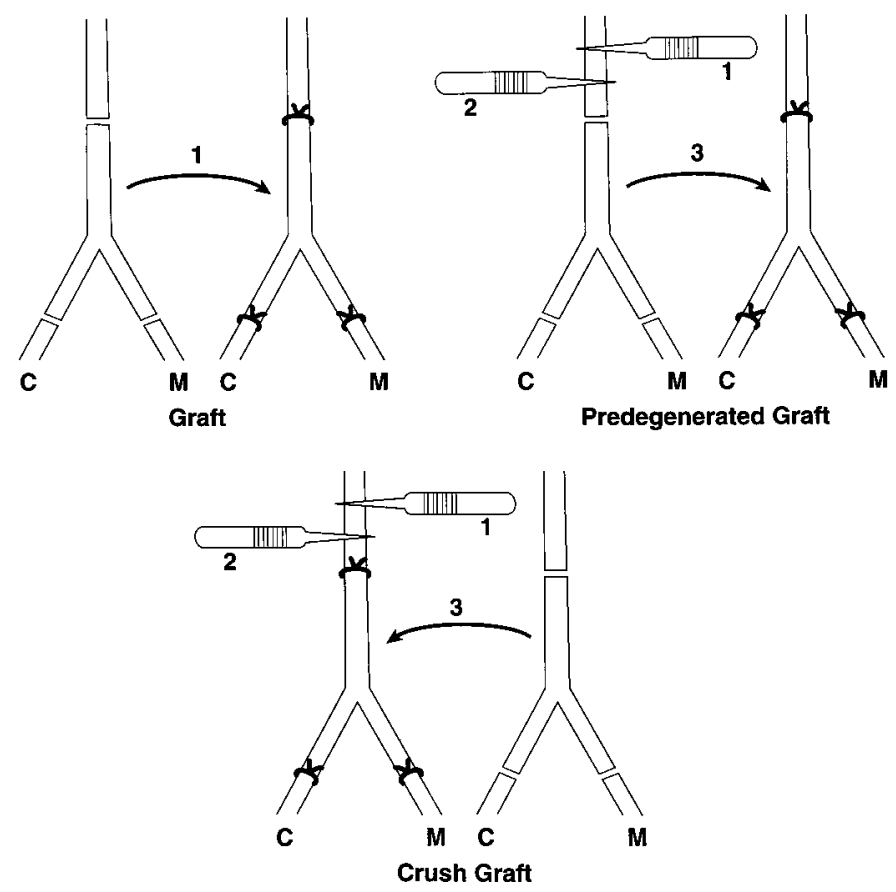

Figure 5. Preparation of grafting experiments. In the graft group, the femoral nerve trunk and branches were excised and sewn into the bed of the opposite femoral nerve, correctly aligning cutaneous, muscle, and iliacus branches. In the predegenerated graft group, the donor segment was predegenerated 4 and 2 weeks before transfer and then transposed to the unoperated limb to be reinnervated by fresh, previously uninjured axons. This sequence was reversed in the crush graft group; crushes were delivered to the recipient nerve to stimulate collateral sprouting, after which a fresh graft was transferred to receive these sprouts. The iliacus branch (not shown) was repaired in all experiments.

mean of $394(n=7)$ after quadriceps axons were misdirected into the cutaneous pathway. The ability of regenerated motor axons to transport HRP is thus independent of the pathway they occupy in this model. Six unoperated proximal femoral nerves contained a mean of 3766 myelinated axons, whereas four that had previously undergone distal crushes contained a mean of 3830. Previous distal crush therefore has no effect on proximal myelinated, and thus motor, axon counts. Proximal crush, in contrast, increased the total number of axons in pure motor preparations from a mean of $866(n=10)$ to a mean of $7055(n=10)$. Because the normal femoral count includes unmyelinated axons, these changes reflect between a fivefold and eightfold increase in the total number of motor axons at the repair site.

\section{DISCUSSION}

The refinement of peripheral connections through collateral pruning has been conserved as a method of specificity generation throughout evolution (Denburg, 1982; Jellies et al., 1987; Baptista and Macagno, 1988; Ferns and Hollyday, 1995; Hollyday and Morgan-Carr, 1995). When mammalian peripheral nerve regenerates, transected axons produce multiple collateral sprouts that advance distally as the "regenerating unit" (Morris et al., 1972). Many of these collaterals survive and become myelinated, and their numbers in regenerating muscle nerve remain elevated by a factor of 1.5-5 for several months (Shawe, 1955; Murray and Edwards, 1982; Jenq and Coggeshall, 1984). Pruning back to a normal myelinated population is not completed in the rat until 2 years after repair (Mackinnon et al., 1991). The pruning hypoth-

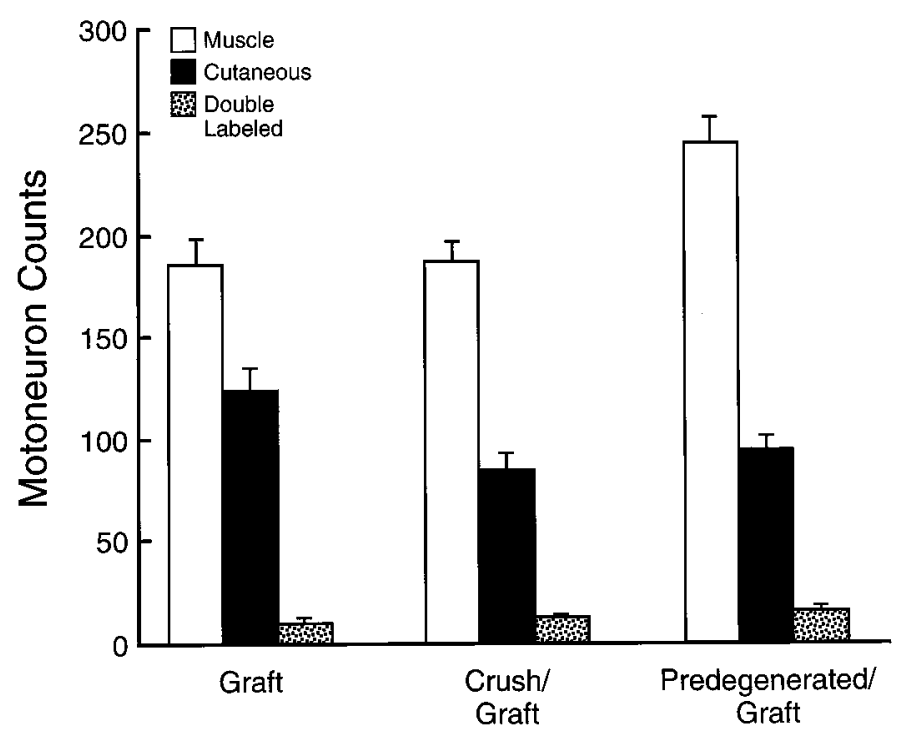

Figure 6. Results of grafting experiments. Each triad of vertical bars represents the means of correct, incorrect, and double-labeled projections from 20 experimental animals. Proximal crush to stimulate regenerative sprouting increased the specificity with which fresh graft was reinnervated, confirming the pruning hypothesis. When predegenerated graft was reinnervated with fresh axons, both specificity (\% M) and the absolute number of motoneurons reinnervating the quadriceps muscle were significantly increased. The results in the predegenerated graft group equal those of crush repair (Fig. 2).

esis suggests that this process may contribute to motor-sensory specificity.

The results of crush repair are consistent with the pruning hypothesis. Crush of the proximal femoral nerve in control animals increased the number of motor axons at the repair site by a factor of five to eight. Analysis of regeneration 3 weeks after crush repair demonstrated an increase in the number of motoneurons projecting collaterals to both cutaneous and muscle nerve, the substrate for collateral pruning. At both 3 and 8 weeks, the percentage of motoneurons projecting correctly to muscle was increased in crush repair compared with repair groups. Specificity was achieved by lowering the number of incorrect cutaneous projections, however, rather than by increasing the number of motoneurons projecting correctly to the quadriceps muscle.

The femoral nerve was chosen for these experiments because the Schwann cell tubes leading to terminal cutaneous and quadriceps muscle branches could be treated as relatively equal "targets" (Brushart, 1993). The nerve was transected proximally, where cutaneous and muscle axons intermingle, to provide regenerating motor axons with equal access to both pathways. The optimal site, identified by axon tracing studies, was proximal to the iliacus muscle branch (Fig. 3). Axons reinnervating the iliacus were excluded from analysis, increasing the rigor of the model by emphasizing the paired distal targets and the numerical importance of incorrect motoneuron projections to skin. However, the possibility that crush repair increased specificity by shunting motor axons from skin to iliacus muscle required a more detailed examination of these projections.

The sequential double-labeling model was introduced to account for all motoneurons regenerating after femoral nerve transection and repair (Fig. 3). Tracer was initially applied to the terminal cutaneous branch and then later to the entire nerve just proximal to the iliacus branch. Incorrect projections to skin were 
thus labeled by both exposures (double-labeled), whereas muscle projections were labeled only once. This technique effectively demonstrated that motoneurons lost from the cutaneous nerve after crush repair had regenerated, presumably reinnervating the iliacus muscle. An increase in iliacus, but not in quadriceps, projections may reflect the proximal location of the iliacus nerve; collaterals reinnervating iliacus muscle would receive trophic support while their siblings are still confined to the distal pathway and are more susceptible to pruning. A situation in which collaterals are pruned from cutaneous nerve and saved in iliacus nerve is consistent with generation of motor-sensory specificity by collateral pruning.

The grafting experiments provide specific support for the pruning hypothesis. In the crush graft group, fresh graft is reinnervated by crushed axons. Predegeneration of the distal stump is thus eliminated as a variable. Neuronal conditioning by sequential crush did not increase specificity in the conditioned repair animals. The significant increase in specificity $(\% \mathrm{M})$ seen in crush graft compared with graft animals must therefore reflect the effects of collateral generation and not those of neuronal conditioning or pathway predegeneration. These collaterals could facilitate exploration of the distal stump and contact recognition of appropriate pathways. However, the frequent observation of equal motoneuron projections to cutaneous and muscle nerve during early regeneration favors the pruning hypothesis over immediate recognition of correct pathways. The failure to increase the number of motoneurons correctly reinnervating the quadriceps muscle parallels the experience with crush repair described above.

Additional aspects of the femoral nerve model may influence the interpretation of our results and thus deserve consideration. Conditioning femoral motoneurons with two distal crushes did not affect the time course or degree of PMR. The conditioning effect can be maximized by superimposing conditioning and test lesions (Bisby, 1985), but the impact of the lesion on conditioning and sprout formation cannot be separated by this approach. In our experiments, the conditioning crushes were $2 \mathrm{~cm}$ distal to the test lesion. Conditioning axotomy a similar or greater distance below the test lesion increases the speed of the slow component $b$ of axoplasmic transport (McQuarrie and Jacob, 1991), enhances subsequent sprout formation (McQuarrie, 1985), and stimulates the expression of GAP 43 in DRG neurons (Chong et al., 1994). It thus seems likely that our motoneurons were effectively conditioned and that conditioning does not affect PMR.

In interpreting our results, it is assumed that motoneurons are labeled, regardless of their distal environment. This assumption is challenged by experiments showing that persistent axotomy decreases labeling of both sensory and motoneurons (Peyronnard et al., 1986). However, it is inappropriate to compare axons in a persistent state of axotomy with those in which axon transport is actively participating in regeneration, regardless of the target. A closer analogy might be earlier experiments in which the femoral cutaneous and muscle pathways were blocked, preventing end organ contact (Brushart, 1993). In this context, PMR was evident, but motoneuron labeling was reduced from both pathways. In control experiments, motor axons forcibly directed to cutaneous or muscle pathways transported HRP with equal effectiveness, confirming the validity of our technique. An additional important aspect of motoneuron quantitation is the technique of counting labeled cells. In these experiments, all labeled motoneurons were counted; nuclei and nucleoli could not be counted reliably, because they were often obscured by dense HRP reaction product.
Frozen sections were cut at $80 \mu \mathrm{m}$ to increase the ratio of section thickness to cell diameter. Total counts of HRP-labeled, FGlabeled, and double-labeled motoneurons from each nerve were multiplied by a correction factor as described by Abercrombie (1946). As the counting progressed, several samples of regenerated motoneurons, all from the femoral motoneuron pool, were found to be similar in shape, size, and orientation. The validity of our group comparisons is therefore uncompromised by systematic bias in counting technique (Saper, 1996; Guillery and Herrup, 1997).

The predegenerated graft group was prepared to evaluate the potential effect of pathway predegeneration on the outcome of crush repair. The dramatic impact of this maneuver was not anticipated. Specificity increased from $58 \% \mathrm{M}$ (graft) to $69 \% \mathrm{M}$ (predegenerated graft), and the mean number of motoneurons reinnervating the quadriceps muscle increased from 186 to 245 . These results were comparable to those of crush repair. Although these are the first experiments to determine the effects of predegeneration on specificity in a nerve repair or graft model, others have examined its broader impact on regeneration. Nerve graft predegeneration was variously found by early investigators to accelerate regeneration (Ramon y Cajal, 1928; Duel, 1933) or to have little or no effect (Huber, 1920; Bentley and Hill, 1936; Young et al., 1940; Sanders and Young, 1942). The results of recent studies are equally varied. In vitro, adult rat DRG neurites grow on predegenerated, but not on normal, nerve sections (Bedi et al., 1992). Embryonic neurites will grow on both but extend longer processes on predegenerated tissue (Agius and Cochard, 1998). In vivo, predegeneration appears to improve the early stages of regeneration (Kerns et al., 1993; Sorenson and Windebank, 1993; Zhao and Kerns, 1994; Hasan et al., 1996). This finding is consistent with the observation that, in the rat model, all grafts are "predegenerated" within a week of nerve transection.

Predegeneration may enhance the specificity of regeneration by several mechanisms. Stimulation of trophic factor production is a leading possibility. The neurotrophic factors BDNF and glialderived neurotropic factor (GDNF) are potential mediators of this effect. Both are upregulated within denervated peripheral nerve (Meyer et al., 1992; Funakoshi et al., 1993; Trupp et al., 1995; Naveilhan et al., 1997) and are transported to parent motoneurons (DiStefano et al., 1992; Yan et al., 1995) that display the appropriate tyrosine kinase receptors TrkB and Ret (Henderson et al., 1993; Koliatsos et al., 1993; Naveilhan et al., 1997). Both promote survival ( $\mathrm{Li}$ et al., 1995; Kishino et al., 1997; Novikov et al., 1997) and phenotypic maintenance (Yan et al., 1994, 1995; Friedman et al., 1995) of axotomized adult motoneurons. Both factors stimulate regeneration of peripheral axons (Utley et al., 1996; Naveilhan et al., 1997). Promotion of motorsensory specificity would require selective upregulation of a trophic factor within muscle, but not cutaneous, pathways. This has been suggested for BDNF on the basis of PCR studies (Rapoza et al., 1994) but has not been examined for GDNF.

Predegeneration could also remove inhibitors of regeneration, allowing axons earlier and more extensive contact with specific pathway markers. Myelin-associated glycoprotein (MAG) has been identified as an inhibitor of axon growth (McKerracher et al., 1994; Mukhopadhyay et al., 1994; Schafer et al., 1996). Clearance of MAG by predegeneration will not in itself enhance regeneration specificity but could improve access to specific pathway markers. An example is the L2 carbohydrate epitope, which predominately labels muscle pathways and preferentially supports 
outgrowth of motor neurites in vitro (Martini et al., 1992). During regeneration, L2 is selectively reexpressed when muscle pathways are reinnervated by motor axons, an interaction that may promote PMR (Martini et al., 1994).

These experiments confirm earlier observations of PMR and demonstrate that collateral pruning contributes to its generation. They have also shifted emphasis from neuron to pathway. A fivefold to eightfold increase in collateral sprouting allowed only modest gains in specificity. Conditioning the neuron with a series of distal crushes had no effect whatsoever. In contrast, predegeneration of the distal pathway dramatically increased both specificity and the absolute number of motoneurons reinnervating the quadriceps muscle. With predegeneration, the outcome of nerve grafting was equal to that of routine nerve repair. Identification of the specific benefits of predegeneration will thus be an important goal of future studies. Further perspective was gained from the sequential double-labeling model. When all motoneurons regenerating in the femoral nerve are accounted for, few are seen to be misdirected to skin: $22 \%$ after repair and only $10 \%$ after crush repair. PMR is thus quite effective in the rat. However, the relatively poor outcome of clinical nerve repair (Wilgis and Brushart, 1993) suggests that PMR may not function as effectively in humans. Primate studies will thus be needed to approximate the effects of PMR in the human and the potential, if any, for clinical improvement through its augmentation.

\section{REFERENCES}

Abercrombie M (1946) Estimation of nuclear population from microtome sections. Anat Rec 94:239-247.

Agius E, Cochard P (1998) Comparison of neurite outgrowth induced by intact and injured sciatic nerves: a confocal and functional analysis. J Neurosci 18:328-338.

Baptista CA, Macagno ER (1988) Modulation of the pattern of axonal projections of a leech motor neuron by ablation or transplantation of its target. Neuron 1:949-962.

Bedi K, Winter J, Berry M, Cohen J (1992) Adult rat dorsal root ganglion neurons extend neurites on predegenerated but not on normal peripheral nerves in vitro. Eur J Neurosci 4:193-200.

Bentley FH, Hill M (1936) Nerve grafting. Br J Surg 24:368-387.

Berod A, Hartman BK, Pujol JF (1981) Importance of fixation in immunohistochemistry: use of formaldehyde solutions at variable $\mathrm{pH}$ for the localization of tyrosine hydroxylase. J Histochem Cytochem 29:844-850.

Bisby M (1985) Enhancement of the conditioning lesion effect in rat sciatic motor axons after superimposition of conditioning and test lesions. Exp Neurol 90:385-394.

Brushart TM (1988) Preferential reinnervation of motor nerves by regenerating motor axons. J Neurosci 8:1026-1031.

Brushart TM (1990) Preferential motor reinnervation: a sequential double-labeling study. Restor Neurol Neurosci 1:281-287.

Brushart TM (1993) Motor axons preferentially reinnervate motor pathways. J Neurosci 13:2730-2738.

Chong M, Reynolds M, Irwin N, Coggeshall R, Emson P, Benowitz L, Woolf C (1994) GAP-43 expression in primary sensory neurons following central axotomy. J Neurosci 14:4375-4384.

Denburg JL (1982) Elimination of inappropriate axonal branches of regenerating cockroach motor neurons as detected by the retrograde transport of horseradish peroxidase conjugated with wheat germ agglutinin. Brain Res 248:1-8.

DiStefano P, Friedman B, Radziejewski C, Alexander C, Boland P, Schick C, Lindsay R, Wiegand S (1992) The neurotrophins BDNF, NT-3, and NGF display distinct patterns of retrograde axonal transport in peripheral and central neurons. Neuron 8:983-993.

Duel AB (1933) History and development of the surgical treatment of facial palsy. Surg Gynecol Obstet 56:382-389.

Ferns MJ, Hollyday M (1995) Chick wing innervation. III. Formation of axon collaterals in developing peripheral nerves. J Comp Neurol 357:272-280.

Friedman B, Kleinfeld D, Ip NY, Verge VMK, Moulton R, Boland P,
Zlotchenko E, Lindsay RM, Liu L (1995) BDNF and NT-4/5 exert neurotrophic influences on injured adult spinal motor neurons. J Neurosci 15:1044-1056.

Funakoshi H, Frisen J, Barbany G, Timmusk T, Zachrisson O, Verge V, Persson H (1993) Differential expression of mRNAs for neurotrophins and their receptors after axotomy of the sciatic nerve. J Cell Biol 123:455-465.

Guillery R, Herrup K (1997) Quantification without pontification: choosing a method for counting objects in sectioned tissues. J Comp Neurol 386:2-7.

Hasan NA, Neumann MM, de Souky MA, So KF, Bedi KS (1996) The influence of predegenerated nerve grafts on axonal regeneration from prelesioned peripheral nerves. J Anat 189:293-302.

Henderson CE, Camu W, Mettling C, Gouin A, Poulsen K, Karihaloo M, Rullamas J, Evans T, McMahon SB, Armanini MP, Berkemeier L, Phillips HS, Rosenthal A (1993) Neurotrophins promote motor neuron survival and are present in embryonic limb bud. Nature 363:266-270.

Hollyday M, Morgan-Carr M (1995) Chick wing innervation. II. Morphology of motor and sensory axons and their growth cones during early development. J Comp Neurol 357:254-271.

Huber C (1920) repair of peripheral nerve injuries. Surg Gynecol Obstet 30:464-471.

Jellies J, Loer C, Kristan W (1987) Morphological changes in leech retzius neurons after target contact during embryogenesis. J Neurosci 7:2618-2629.

Jenq C-B, Coggeshall RE (1984) Regeneration of axons in tributary nerves. Brain Res 310:107-121.

Kerns JM, Danielsen N, Holmquist B, Kanje M, Lundborg G (1993) The influence of predegeneration on regeneration through peripheral nerve grafts in the rat. Exp Neurol 122:28-36.

Kishino A, Ishige Y, Tatsuno T, Nakayama C, Noguchi H (1997) BDNF prevents and reverses adult rat motor neuron degeneration and induces axonal outgrowth. Exp Neurol 144:273-286.

Koliatsos V, Clatterbuck R, Winslow J, Cayouette M, Price D (1993) Evidence that brain-derived neurotrophic factor is a trophic factor for motor neurons in vivo. Neuron 10:359-367.

Li LX, Wu WT, Lin LFH, Lei M, Oppenheim RW, Houenou LJ (1995) Rescue of adult mouse motoneurons from injury-induced cell death by glial cell line-derived neurotrophic factor. Proc Natl Acad Sci USA 92:9771-9775.

Mackinnon S, Dellon L, O’Brien J (1991) Changes in nerve fiber numbers distal to a nerve repair in the rat sciatic nerve model. Muscle Nerve 14:1116-1122.

Madison RD, Archibald SJ, Brushart TM (1996) Reinnervation accuracy of the rat femoral nerve by motor and sensory neurons. J Neurosci 16:5698-5703.

Martini R, Xin Y, Schmitz B, Schachner M (1992) The L2/HNK-1 carbohydrate epitope is involved in the preferential outgrowth of motor neurons on ventral roots and motor nerves. Eur J Neurosci 4:628-639.

Martini R, Schachner M, Brushart TM (1994) The L2/HNK-1 carbohydrate is preferentially expressed by previously motor axon-associated Schwann cells in reinnervated peripheral nerves. J Neurosci 14:7180-7191.

McKerracher L, David S, Jackson DL, Kottis V, Dunn RJ, Braun PE (1994) Identification of myelin-associated glycoprotein as a major myelin-derived inhibitor of neurite growth. Neuron 13:805-811.

McQuarrie IG (1985) Effect of a conditioning lesion on axonal sprout formation at nodes of ranvier. J Comp Neurol 231:239-249.

McQuarrie IG, Jacob J (1991) Conditioning nerve crush accelerates cytoskeletal protein transport in sprouts that form after a subsequent crush. J Comp Neurol 305:139-147.

Mesulam M-M (1982) Tracing neural connections with horseradish peroxidase. New York: Wiley.

Meyer M, Matsuoka I, Wetmore C, Olson L, Thoenen H (1992) Enhanced synthesis of brain-derived neurotrophic factor in the lesioned peripheral nerve: different mechanisms are responsible for the regulation of BDNF and NGF mRNA. J Cell Biol 119:45-54.

Morris JH, Hudson AR, Weddell G (1972) A study of degeneration and regeneration in the divided rat sciatic nerve based on electron microscopy II. The development of the "regenerating unit". Z Zellforsch Mikrosk Anat 124:103-130.

Mukhopadhyay G, Doherty P, Walsh FS, Crocker PR, Filbin MT (1994) A novel role for myelin-associated glycoprotein as an inhibitor of axonal regeneration. Neuron 13:757-767. 
Murray M, Edwards MA (1982) A quantitative study of the reinnervation of the goldfish optic tectum following optic nerve crush. J Comp Neurol 209:363-373.

Naveilhan P, ElShamy VM, Emfors P (1997) Differential regulation of mRNAs for GDNF and its receptors Ret and GDNFR after sciatic nerve lesion in the mouse. Eur J Neurosci 9:1450-1460.

Neumann CM, Brushart TM, Gordon T (1996) Increasing the specificity of regenerating motor nerves. Soc Neurosc Abstr 22:1487.

Novikov L, Novikova L, Kellerth JO (1997) Brain-derived neurotrophic factor promotes axonal regeneration and long-term survival of adult rat spinal motoneurons in vivo. Neuroscience 79:765-774.

Peyronnard J, Charron L, Lavoie J, Messier J (1986) Differences in horseradish peroxidase labeling of sensory, motor and sympathetic neurons following chronic axotomy of the rat sural nerve. Brain Res 364:137-150.

Ramon y Cajal S (1928) Degeneration and regeneration of the nervous system. London: Oxford UP.

Rapoza MP, Aldous MD, Archibald SJ, Rohwer RJ, Madison RD (1994) BDNF levels increase in the terminal motor branch following lesions of the parent femoral nerve: a competitive PCR study. Soc Neurosc Abstr 20:1500.

Sanders FK, Young JZ (1942) The degeneration and re-innervation of grafted nerves. J Anat 76:143-170.

Saper C (1996) Any way you cut it: a new journal policy for the use of unbiased counting methods. J Comp Neurol 364:5.

Schafer M, Fruttiger M, Montag D, Schachner M, Martini R (1996) Disruption of the gene for the myelin-associated glycoprotein improves axonal regrowth along myelin in C57BL/Wld mice. Neuron 16:1107-1113.
Schmued LC, Fallon J (1986) Fluoro-gold, a new fluorescent retrograde axonal tracer with numerous unique properties. Brain Res 377:147-154.

Shawe GDH (1955) On the number of branches formed by regenerating nerve-fibers. Br J Surg 42:474-488.

Sorenson E, Windebank A (1993) Relative importance of basement membrane and soluble growth factors in delayed and immediate regeneration of rat sciatic nerve. J Neuropathol 52:216-222.

Trupp M, Ryden M, Jornvall H, Funakoshi H, Timmusk T, Arenas E, Ibanez C (1995) Peripheral expression and biological activities of GDNF, a new neurotrophic factor for avian and mammalian peripheral neurons. J Cell Biol 130:137-148.

Utley DS, Lewin SL, Cheng ET, Verity AN, Sierra D, Terris DJ (1996) Brain-derived neurotrophic factor and collagen tubulization enhance functional recovery after peripheral nerve transection and repair. Arch Otolaryngol Head Neck Surg 122:407-413.

Wilgis EFS, Brushart TM (1993) Nerve repair and grafting. In: Operative Hand Surgery, Vol 3 (Green DP, ed), pp 1315-1340. New York: Churchill-Livingstone.

Yan Q, Matheson C, Lopez OT, Miller JA (1994) The biological responses of axotomized adult motoneurons to brain-derived neurotrophic factor. J Neurosci 14:5281-5291.

Yan Q, Matheson C, Lopez O (1995) In vivo neurotrophic effects of GDNF on neonatal and adult facial motoneurons. Nature 373:341-344.

Young JZ, Holmes W, Sanders FK (1940) Nerve regeneration: importance of peripheral stump and the value of nerve grafts. Lancet 2:128-130.

Zhao Q, Kerns JM (1994) Effects of predegeneration on nerve regeneration through silicone Y-chambers. Brain Res 633:97-104. 